\title{
Transposition
}

Musique et Sciences Sociales

$4 \mid 2014$

Musique et conflits armés après 1945

\section{Márta Grabócz (dir.), Les opéras de Peter Eötvös entre Orient et Occident}

Paris, Éditions des archives contemporaines, 2012, 174 p.

\section{Béatrice Ramaut-Chevassus}

\section{OpenEdition}

\section{Journals}

Édition électronique

URL : http://journals.openedition.org/transposition/499

DOI : 10.4000/transposition.499

ISSN : 2110-6134

Éditeur

CRAL - Centre de recherche sur les arts et le langage

\section{Référence électronique}

Béatrice Ramaut-Chevassus, « Márta Grabócz (dir.), Les opéras de Peter Eötvös entre Orient et Occident », Transposition [En ligne], 4 | 2014, mis en ligne le 15 juillet 2014, consulté le 22 septembre 2020. URL : http://journals.openedition.org/transposition/499; DOI : https://doi.org/10.4000/transposition. 499

Ce document a été généré automatiquement le 22 septembre 2020.

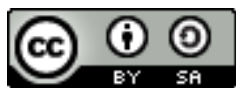

La revue Transposition est mise à disposition selon les termes de la Licence Creative Commons Attribution - Partage dans les Mêmes Conditions 4.0 International. 


\title{
Márta Grabócz (dir.), Les opéras de Peter Eötvös entre Orient et Occident
}

Paris, Éditions des archives contemporaines, 2012, 174 p.

\author{
Béatrice Ramaut-Chevassus
}

\section{RÉFÉRENCE}

Márta Grabócz (dir.), Les opéras de Peter Eötvös entre Orient et Occident, Paris, Éditions des archives contemporaines, 2012, $174 \mathrm{p}$.

1 La musicologie de langue française a donné en 2012 deux ouvrages importants pour la connaissance des opéras du compositeur Peter Eötvös : d'une part, le collectif dirigé par Márta Grabócz Les opéras de Peter Eötvös entre Orient et Occident et d'autre part, les Entretiens autour des cinq premiers opéras de Peter Eötvös d'Aurore Rivals ${ }^{1}$. Les deux livres ne sont pas étrangers l'un à l'autre puisque c'est à Aurore Rivals qu'a été confié le premier chapitre du livre de Márta Grabócz. Mentionnons que, déjà en 2007, un ouvrage plus généraliste avait été consacré au compositeur hongrois à la suite d'un colloque tenu en sa présence à la Hochschule für Musik de Bâle ${ }^{2}$. Publié en allemand sous la direction de Michael Kunkel, il contenait divers entretiens, une section intitulée « Kontext» comprenant quatre articles, une autre en comprenant cinq sur diverses œuvres allant de Kosmos à Trois sœurs, et la transcription d'une table ronde sous le titre «Musik aus einem Guss ».

Le succès du Hongrois Peter Eötvös né en 1944, longtemps connu comme remarquable chef d'orchestre plus que comme compositeur malgré un catalogue déjà très vaste ${ }^{3}$, a été fulgurant après la création de sa première œuvre lyrique il y a quinze ans. Si les six opéras qu'il a composés à ce jour sont entrés au répertoire en Europe et en Amérique du Nord, il faut souligner que les quatre premiers ont un lien particulier avec la France où ils ont été commandés et créés : Trois Sœurs (Opéra de Lyon, le 13 mars 1998, en russe); Le Balcon (Festival d'Aix en Provence, le 5 juillet 2002, en français); Angels in America (Théâtre du Chatelet, le 23 novembre 2004, en américain); Lady Sarashina 
(Opéra de Lyon, le 4 mars 2008, en anglais). Trois opéras sont par ailleurs actuellement en commande (pour Vienne, Francfort et New York ${ }^{4}$ ), c'est dire la pertinence qu'il y a à s'intéresser à l'œuvre scénique d'un compositeur si fécond.

3 Márta Grabócz, membre de l'IUF, responsable de l'axe de recherche "Approches sémiotiques et esthétiques de l'acte musical» du LABEX GREAM (Université de Strasbourg), s'entoure pour cette publication de cinq musicologues ayant tous déjà signé des travaux sur les opéras d'Eötvös. Alors que le titre ne le précise pas, quatre opéras seulement sont présentés dans ce volume. Il ne s'agit pas des quatre premiers mais de Trois Soeurs, Angels in America, Lady Sarashina et Love and Other Demons (Festival de Glyndebourne, 10 août 2008, anglais, espagnol, latin, yoruba). Après une introduction de Márta Grabócz, le chapitre d'Aurore Rivals traite de "La construction des livrets ", puis quatre chapitres - que Márta Grabócz qualifie à juste titre d'essais (p. 5) - abordent chacun des opéras : Marie Laviéville-Angelier, «Trois scurs ou l'art de l'épure »; Kristina Megyeri, "Les projections du présent dans Angels in America»; François-Gildas Tual, «Lady Sarashina "À la recherche d'un monde perdu" »; JeanFrançois Boukobza, "Love and other demons un théatre pour l'intelligence ». L'ouvrage est complété par un "catalogue des opéras et genres apparentés ", des "orientations bibliographiques, discographiques et filmiques ", ainsi que les "notices biographiques des auteurs ».

Alors que l'œuvre de Peter Eötvös, fondé conjointement sur un attachement à la musique comme langue et à la langue comme musique, est tout entier une célébration de la voix humaine, Márta Grabócz aborde avec pertinence son introduction (p. 1-6) par les thèmes contenus dans les livrets, soulignant leur richesse et la diversité de leur provenance géographique. Elle rappelle que la Hongrie a été qualifiée par l'un de ses poètes, Endre Ady, "de "pays-bac" (pays navette) oscillant sans cesse entre Orient et Occident ", expliquant ainsi l'aisance avec laquelle Eötvös passe de l'un à l'autre et le titre du livre. L'universalité de la musique de ce «citoyen du monde ${ }^{5}$ » s'expliqueraitelle également ainsi? Des propos recueillis par Marie Lavieville, repris par FrançoisGildas Tual (p.102) rendent compte avec plus d'acuité encore d'une convergence entre Orient et Occident dans l'identité hongroise même. Eötvös dit en avoir eu la révélation lors de son voyage au Japon avec Stockhausen en 1970: «J'y ai trouvé une certaine connexion avec la mentalité des paysans hongrois. Leur comportement est très proche, il semble y avoir une parenté qui m'a encore plus frappé lorsque je suis retourné en Hongrie. C'est peut-être parce que nous avons une racine commune, nos ancêtres venaient de Mongolie... ». Si Eötvös est un passeur entre différents pays, il l'est aussi entre différentes langues et différentes époques. Márta Grabócz mentionne ensuite des points communs qui relieraient les livrets des quatre opéras, malgré leur diversité. Elle en discerne deux. Les livrets traiteraient tous «d'un moment relativement chaotique de l'histoire, d'une crise survenant pour clore une époque d'équilibre ou d'un âge d'or en voie de disparition» (p. 2) ; ils feraient tous appel à "La présence des forces surnaturelles, cathartiques ou transcendantes » (p. 3).

5 Le chapitre sur « La construction des livrets » (p. 7-36) revient donc à Aurore Rivals auteure de Peter Eötvös, le passeur d'un savoir renouvelé. Pour une archéologie de la composition ou dix ans d'opéra (thèse soutenue à Paris-Sorbonne, 2010). Elle montre que le travail musical commence dès la fabrication du livret, «ce lien incontournable entre littérature et musique»(p.7), mais aussi ce lieu où se reflètent les contraintes formelles, administratives et économiques de la commande quant à l'effectif orchestral, 
au nombre de chanteurs et à leurs tessitures ou encore quant à la durée de l'œuvre. "L'aspect pragmatique prédomine dans la démarche sélective » du compositeur-chef d'orchestre. Quant au choix des auteurs et des textes - Anton Tchekhov pour Trois Sœurs, Tony Kushner pour Angels in America, un nikki millénaire pour Lady Sarashina, Gabriel García Márquez pour Love and other demons - Aurore Rivals affirme qu'« [Eötvös] choisit le texte en fonction des caractéristiques de la maison d'opéra pour laquelle il écrit : la portée sémantique et symbolique du texte doit s'accorder au public, au profil et au style de chaque institution »(p. 7-8). L'auteure livre les résultats de ses analyses en des tableaux synthétiques très clairs qui donnent à voir tout au long de l'article l'organisation des livrets et les prélèvements effectués sur les œuvres littéraires originales. Ces dernières incarneraient respectivement « Le temps de la désagrégation » pour Trois sœeurs, «Le théâtre de l'apocalypse » pour Angels in America, « La vie-fiction » pour Lady Sarashina, "Le roman-fantasme " pour Love and other demons. Pour Aurore Rivals, «Peter Eötvös hisse au statut de mythe des hommes et des femmes ordinaires ou extraordinaires ». Ainsi, la question du sujet des livrets rejoint celle de la transmission des opéras et Aurore Rivals en vient à interpréter leur réception. Par ses opéras, Eötvös ferait un «don du mythe aux générations futures » pour " donner l'envie de jouer sa musique et de la transmettre» (p. 36). L'auteure suggère en outre, dans le cas de Trois sœurs, que "l'ouvrage lyrique serait la "représentation intemporelle" de la pièce de théâtre qui se déroule chronologiquement ", proche de l'intentio augustinien (p.17). Dans cet excellent chapitre, deux points mériteraient précision. Lorsqu'Aurore Rivals constate (p. 8) l'absence de tension téléologique dans les quatre livrets, et affirme pour cela que « le vocable "acte" ne figure dans aucun des quatre opéras ", préférant ceux de "séquence " ou de "partie", n'y a-t-il pas contradiction avec l'information exacte donnée p. 168 que Love and other demons est en deux actes? Par ailleurs, si le compositeur participe activement à l'élaboration des livrets, il ne s'en dit cependant jamais le seul auteur. D'après les partitions, C.H. Henneberg et Peter Eötvös sont coauteurs de celui de Trois sœurs, Mari Mezei - femme de Peter Eötvös - est auteure de ceux d'Angels in America et de Sarashina, et Kornél Hamvai auteur de celui de Love and other demons. N'est-ce pas inexact d'écrire qu'Eötvös «confectionne lui-même [les livrets] aux côtés de son épouse » (p. 32), sans même jamais nommer cette dernière ?

6 Alors que les publications musicologiques sont déjà nombreuses sur l'opéra inaugural Trois sœurs, Marie Laviéville - auteure de L'Esthétique de Peter Eötvös, une dramaturgie de la relation (thèse soutenue à Lille en 2010) - en propose une analyse transversale efficace et minutieuse, étayée par trente exemples musicaux, huit schémas synthétiques et des fragments d'entretiens avec le compositeur (p. 37-75). Des possibilités dramaturgiques offertes par le "principe d'association systématique donnant à chaque personnage un avatar instrumental ", à la "recherche approfondie en matière de typologie vocale " dans cet opéra où le trio des sœurs est incarné par trois voix d'hommes, en passant par les "clés de compréhension harmonique " et les "sonorités " mises en place dès le prologue, ou encore par l'exposé des "réitérations musicales à fonction symbolique " que sont entre autres les motifs, Marie Laviéville recense les moyens musicaux de la cohésion dramaturgique de cet opéra écrit sur un livret russe avec la collaboration du chorégraphe japonais Ushio Amagatsu grâce auquel Peter Eötvös dit avoir surmonté sa "peur du geste opératique»(p.39). Sont ainsi abordés successivement les points suivants : "Une caractérisation instrumentale », «Une vocalité individualisée », «Un matériau générateur et signifiant », «La répétition au service de la dramaturgie », « Un diptyque amoureux, le cycle des déclamations et des adieux » et « La postérité musicale 
de Trois sœurs" dans des œuvres orchestrales ou de musique de chambre du compositeur lui-même.

$7 \quad$ Kristina Megyeri - auteure de Dramaturgie(s) des anges : temporalité, identité et la place $d u$ corps dans Angels in America de Peter Eötvös (master 2, Paris-8, 2006) - restreint son approche d'Angels in America à la question de la temporalité et de la "présence», au sens de projection du temps présent (p. 77-95). Selon Kristina Megyeri, les qualités des différents temps «présents » vécus par les protagonistes sont liées aux rapports qu'ils établissent avec l'Ange. Elle se donne pour but de «saisir brièvement les relations du terme présent avec les vastes possibilités d'imaginaire qui en résultent, puis [d']essayer d'en catégoriser les modes de perception reliés aux réalisations musicales » (p. 78). Elle oppose un présent vivant à un présent totalement régi et déformé par le passé (id.). Les références théoriques de Kristina Megyeri ne sont pas toujours précises pour le lecteur. Celle à Walter Benjamin (Sur le concept d'Histoire ${ }^{6}$ ) ne se fait explicite que tardivement, et Kristina Megyeri n'hésite pas à s'en écarter, par exemple quand elle parle d'un « temps en boucle » correspondant à un "état où le passé ne serait pas "digéré" ». Elle pose que «la ligne philosophico-dramaturgique de la thématique se différencie entre les deux actes $^{7} »$ (p. 82). Le premier serait une "introduction au présent transcendant», le second une "démythification du transcendant». Kristina Megyeri opère une distinction entre le présent intense, le présent transcendant, le présent terne, et le présent ruiné. Dans cette logique, le premier acte serait celui du présent subjectif ou encore du présent imparfait marqué à la fois par la découverte que Prior fait de sa maladie - il est atteint du sida - et par la Voix de l'Ange qu'il commence à entendre (voir p. 86 le tableau récapitulant les apparitions de l'Ange). Le second acte désenchanterait en revanche ces visions et « se focalise[rait] sur une vision utopique de l'à-présent benjaminien» (p. 82). L'apparition de l'Ange est désacralisée. Prior affirme violemment sa simple et forte volonté de vivre. Il rend le Livre des Prophètes et refuse sa vocation. Saluant la grande ouverture de Peter Eötvös sur son temps, Kristina Megyeri en vient à conclure que «la musique d'Angels in America se situe quelque part entre la musique de film, la musique de scène, l'opéra romantique et la comédie musicale " (p. 94). Cet essai ouvre des perspectives intéressantes sur l'opéra dont le succès le plus grand a été remporté sur le continent nord-américain, mais il laisse dans l'ombre des aspects musicaux remarquables, malgré les huit extraits de partition donnés en exemple.

8 François-Gildas Tual - auteur de « Les scènes rêvées de Peter Eötvös, actions musicales et théâtre de l'inaction ", Les relations musique-théâtre: du désir au modèle, l'Harmattan, 2010, p. 175-186 - signe l'étude sur Lady Sarashina (p. 97-125). Son texte est accompagné de huit exemples musicaux et de huit figures synthétisant des points d'analyse dont certaines sont fondées sur des documents sources transmis par le compositeur luimême. François-Gildas Tual s'attache à replacer les opéras d'Eötvös dans une histoire du genre allant de Monteverdi à Puccini, de Britten à Hosokawa. Comme l'ensemble des contributeurs de ce volume, il s'intéresse d'abord au livret, le rapprochant par exemple de celui de Da Gelo a gelo de Salvatore Sciarrino (2006) également fondé sur le journal d'une Japonaise du XI $\mathrm{XI}^{\mathrm{e}}$ siècle, le comparant aussi à d'autres opéras d'Eötvös dont Die Tragödie des Teufels (Munich, février 2010) pour pointer « une même façon d'édifier le théâtre sur des rôles qui sont moins des personnages que des idées de personnages " (p.97). François-Gildas Tual évoque ensuite les "Inspirations japonaises» du compositeur dont As I Crossed A Bridge of Dreams que l'opéra entier remploie. Il analyse les structures littéraires et musicales des neuf scènes de l'opéra en référence aux 
"Songes, souvenirs et contemplations", montrant comment "la dramaturgie, se détournant du déroulement chronologique, fait l'expérience de la lenteur, se soumet aux sauts prospectifs ou rétrospectifs, jusqu'à pouvoir concentrer toute une vie d'attente dans un court instant de prospection" (p. 105). Il s'intéresse au "Dédoublement", catégorie qui concerne aussi bien les situations dramatiques, le matériau musical, les costumes, les rôles, que les liens entre voix et instruments. Quant à la "Conscience du temps", tissée dans le livret par un lien fort à la nature, aux saisons, aux heures du jour, mais aussi au passé précis ou imprécis de Sarashina, François-Gildas Tual montre qu'elle est mise en œuvre musicalement à la fois par une grande maîtrise des cheminements harmoniques subtils, par une gestion de l'espace et par un contact constant entre sons naturels et sons artificiels. Si François-Gildas Tual clôt sa contribution avec des "Remarques d'un spectateur (Miroirs) », ce ne sont pas les siennes, mais celles d'Eötvös lui-même: "Je suis compositeur mais je me considère avant tout comme un spectateur et je cherche donc à faire quelque chose qui fonctionne pour les auditeurs » (p. 125).

Jean-François Boukobza - auteur du "Guide d'écoute ", Trois Sceurs, L'Avant Scène Opéra, $\mathrm{n}^{\circ}$ 204, septembre-octobre 2001 - «entend, modestement, proposer à la fois une découverte de [Love and other demons] et offrir une première tentative de réponse » à la "question des moyens mis en jeu " pour "marier le fameux "réalisme magique" de Gabriel García Márquez avec le goût de Peter Eötvös pour un théâtre psychologique mêlant intériorisation et distanciation ". Il réussit pleinement sur les deux tableaux en donnant à lire une étude très bien documentée, illustrée de treize exemples musicaux, de quatre tableaux et deux annexes (p. 127-164). Après avoir passé la nouvelle de García Márquez au triple prisme de la "polysémie ", de la «luxuriance » et de «l'amour... » (p.127-128), Jean-François Boukobza aborde la confection du livret c'est-à-dire la restructuration, la stylisation et l'interprétation du texte initial. $\mathrm{Si}$ « le texte a changé constamment jusqu'à la phase même de la composition musicale » (p. 133), « l'ensemble emprunte sa conception au cinéma » et "aux techniques de montage rapide ». Alors que la grande forme est rythmée par des retours de motifs (le cryptogramme de Sierva Maria, le motif du rêve, le chant d'oiseau, la prière en latin, le poème de G. de la Vega en espagnol), l'enjeu de l'analyse de Jean-François Boukobza est cependant de montrer que «le risque de rhapsodie est évité par un travail organique extrêmement élaboré » (p.132), un travail qu'il nomme une "Unité de l'inconscient auditif» (p. 137). JeanFrançois Boukobza montre que le cryptogramme, "chargé d'une dimension spéculative ", génère le tissu harmonique entier de la pièce à l'exception de rares textures ou épisodes. Sa mélodie fait office de réservoir harmonique/intervallique (p.137-140). Il montre que la recherche d'homogénéité relève aussi d'une volonté explicite d'euphonie et de synesthésie. Le ton gris-bleu qui domine la mise en scène entre volontairement en résonance avec les sonorités argentines qui enveloppent l'opéra. La dernière partie de l'article confronte l'écriture spécifique du compositeur aux formes historiques du genre, respectées et transgressées : récitatif, air, air de folie, duo amoureux. Jean-François Boukobza aboutit enfin à l'idée que cet opéra est «Un théâtre du silence » (p. 163), dans lequel la musique donne à entendre - pour les oreilles et pour l'esprit - sans nul besoin d'emphase les non-dits du livret, suscitant « une lecture polysémique en faisant appel à l'intelligence et à l'instinct du spectateur ».

10 Les essais originaux que rassemble ce livre contribuent donc vraiment à la connaissance d'«Un théâtre dont il est difficile de faire l'économie aujourd'hui » 
(p. 164). Le lecteur-auditeur reste dans l'attente d'un second volume qui viendrait compléter utilement celui-ci en présentant d'autres œuvres lyriques de Peter Eötvös.

\section{NOTES}

1. Paris, Éditions Aedam Musicae, 2012.

2. KUNKEL, Michael (éd.), Kosmoi, Peter Eötvös an der Hochschule für Musik der Musik-Akademie der Stadt Basel - Schriften, Gespräche, Dokumente, Saarbrücken, PFAU-Verlag, 2007, 332 p.

3. Lire à titre d'exemple l'article déjà ancien de ALBÈRA, Philippe, « Entretien avec Peter Eötvös », in Contrechamps-Festival d'Automne, Paris, 1989, p. 120-125. Alors qu'Albèra interroge Eötvös sur les bons compositeurs de sa génération, Eötvös répond seulement en tant que chez d'orchestre : «chaque année, je lis environ mille partitions. Dans une composition, je recherche par exemple: une conception sonore précise, une capacité formelle, une connaissance des instruments, une force «dramatique» du discours, une conception de l'articulation. Ces deux dernières qualités manquent la plupart du temps".

4. Voir http://eotvospeter.com/commissions

5. Article de HALASZ, Peter, "Peter Eötvös, un Sekler citoyen du monde », Théâtre du Châtelet, programme de salle, 2004, p. 96-101.

6. BENJAMIN, Walter, Sur le concept d'histoire, Euvres III, Paris, Gallimard, coll. "Folio Essais ", 2000, p. 427-444. Trad. française M. de Gandillac, R. Rochlitz et P. Rusch de « Ûber der Begriff der Geschichte ", Gesammelte Schriften, Francfort-sur-le-Main, Suhrkamp Verlag, 1974, t. I (2), p. 691-704.

7. Remarquons qu'ici, Kristina Megyeri introduit de façon inexacte des "actes " dans Angels in America là où il ne s'agit assurément que de « parties ». 